\title{
Contingency Constrained Optimal Power Flow by Hybrid Optimization Technique Using FACTS Device
}

\author{
K.Srilakshmi Lavanya ${ }^{1}$, C.Kumar ${ }^{2}$, Dr.Ch.Padmanabha Raju ${ }^{3}$ \\ ${ }^{I}$ (EEE Department, Prasad V. Potluri Siddhartha Institute of Technology, India) \\ ${ }^{2}$ (Associate professor, EEE Department, Prasad V. Potluri Siddhartha Institute of Technology, India) \\ ${ }^{3}$ (Professor, EEE Department, Prasad V. Potluri Siddhartha Institute of Technology, India)
}

\begin{abstract}
Optimization algorithms are very important for the Optimal Power Flow (OPF). They could be divided into two classes: traditional local search methods and heuristic global ones. Interior point (IP) algorithm has been known as one of the most prominent and fastest method, but its local exploitation characteristic leads to the fact that it could be easily trapped by local optimum. However, heuristic methods such as Adaptive Particle Swarm Optimization (APSO) possess better convergence quality although their convergence speed is not good enough.

This paper presents an Interior point method (IPM) and Adaptive particle swarm optimization (APSO) based hybrid method to solve the contingency constrained optimal power flow (OPF) in power systems incorporated with Flexible Ac Transmission Systems (FACTS). A versatile FACTS device TCPS (Thyristor controlled phase shifter) is considered. In the solution process APSO coupled with IPM to keep the power flows within their security limits. This Hybrid OPF algorithm with TCPS effectively relieves line flow violations under different single line contingencies. Severity Index is used as an objective function to be minimized to improve the security of the power system. The efficiency of proposed algorithm is illustrated by carrying simulation studies on IEEE 30 bus system .This analysis reveals that the proposed algorithm is quite simple and efficient for solving OPF problem.
\end{abstract}

Keywords: Adaptive Particle Swarm Optimization, Contingencies, Flexible AC Transmission, Interior Point Method and Severity Index.

\section{Introduction}

With the ever increasing complexities in power systems across the globe and the growing need to provide stable, secure, controlled, economic and high quality electric power, especially in today's deregulated environment, it is envisaged that Flexible AC Transmission System (FACTS) devices are going to play a critical role in power transmission systems. These devices enhance the stability of the power system both with their fast control characteristics and continuous compensating capability. A FACTS device can control power flow and increase the transmission capacity effectively. Several techniques have been proposed in the past for the adjustment of controllable series capacitor to alleviate line overloads [1]-[3].

The main method uses the model of series capacitor or phase shifter in power flow program. It is possible to alleviate power flow violation and enhance power system security in an electrical power system by use of phase shifter. However, it is well known that the phase shifter adjustment under given contingencies may fail to yield convergence. Thus, optimal power flow (OPF) with phase shifter is a good choice.

The goal of optimal power flow is to determine optimal control variables and quantities for efficient Power system planning and operation. Over the last three decades, OPF solution algorithms used different mathematical programming techniques [4]-[8]. Among those were sequential linear programming (SLP), quadratic programming $(\mathrm{QP})$ and Newton based nonlinear programming methods [5]. In the late 1980s, interest in applying interior point methods (IPM) to power systems applications has increased due to the enhanced performance and convergence properties of the IPM [6]- [8].

A new category of classical optimization tools has emerged to cope with some of the traditional optimization algorithms' shortcomings [9]. The main modern optimization techniques are genetic algorithm (GA) [10], [11], evolutionary programming (EP) [12], artificial neural network (ANN), simulated annealing (SA), Ant Colony optimization (ACO). Recently, a new evolutionary computation technique, called particle swarm optimization (PSO), has been proposed and introduced [13]-[15]. This technique combines social psychology principles in socio-cognition human agents and evolutionary computations. PSO has been motivated by the behavior of organisms such as fish schooling and bird flocking. Generally, PSO is characterized as simple in concept, easy to implement, and computationally efficient. Unlike the other heuristic techniques, PSO has a flexible and well-balanced mechanism to enhance and adapt to the global and local exploration abilities [11].

This paper presents an IPM-APSO (Adaptive Particle Swarm Optimization) integrated hybrid approach with TCPS for removing line overloads under single line contingencies. The organization of this paper is as 
follows. Section II describes TCPS model. Section III contains general formulation of OPF.Section IV describes overview of Particle Swarm Optimization. Section V provides an overview of algorithm. Section VI presents the results of the simulations conducted using the proposed approach. Finally Section VII presents the conclusion.

\section{Thyristor Controlled Phase Shifter Model}

Thyristor Controlled Phase shifter with quadrature voltage injection controls the active power via phase adjustment $\varphi$. The modeling of this device is based on the idea of varying phase angle. The phase angle value of the TCPS is changed automatically to restrict the branch power at pre-defined value. With the help of NR method, the phase angle of the TCPS is gritty efficiently. The TCPS equivalent circuit is shown in Fig.1.

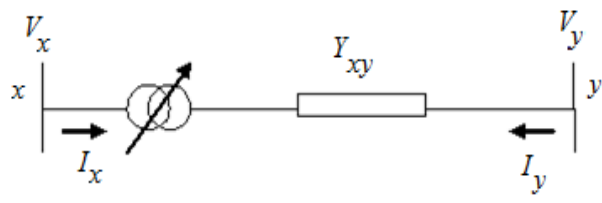

Fig.1 Equivalent circuit of TCPS

From the Fig.1, the current equations at node $\mathrm{x}$ and $\mathrm{y}$ represented in the matrix form is:

$$
\left[\begin{array}{l}
\mathbf{I}_{\mathbf{x}} \\
\mathbf{I}_{\mathrm{y}}
\end{array}\right]=\left[\begin{array}{cc}
\mathbf{Y} & -\mathbf{Y}(\cos \varphi+\mathbf{j} \sin \varphi) \\
-\mathbf{Y}(\cos \varphi-\mathbf{j} \sin \varphi) & \mathbf{Y}
\end{array}\right]\left[\begin{array}{l}
\mathbf{V}_{\mathrm{x}} \\
\mathbf{V}_{\mathrm{y}}
\end{array}\right]
$$

Based on equation (1), equations for the nodal power injections of the thyristor controlled phase shifter, where $\varphi$ is allowed to vary within design rating values $\left(\varphi_{\min }<\varphi<\varphi_{\max }\right)$, are as follows:

$$
\begin{aligned}
& P_{x}=V_{x}^{2} G_{x x}+V_{x} V_{y}\left[G_{x y} \cos \left(\delta_{x}-\delta_{y}\right)+B_{x y} \sin \left(\delta_{x}-\delta_{y}\right)\right] \\
& Q_{x}=-V_{x}^{2} B_{x x}+V_{x} V_{y}\left[G_{x y} \sin \left(\delta_{x}-\delta_{y}\right)-B_{x y} \cos \left(\delta_{x}-\delta_{y}\right)\right] \\
& P_{y}=V_{y}^{2} G_{y y}+V_{y} V_{x}\left[G_{y x} \cos \left(\delta_{y}-\delta_{x}\right)+B_{y x} \sin \left(\delta_{y}-\delta_{x}\right)\right] \\
& Q_{y}=-V_{y}^{2} B_{y y}+V_{y} V_{x}\left[G_{y x} \sin \left(\delta_{y}-\delta_{x}\right)-B_{y x} \cos \left(\delta_{y}-\delta_{x}\right)\right]
\end{aligned}
$$

Where

$$
\left.\begin{array}{c}
Y_{x x}=Y_{y y}=Y \\
Y_{x y}=-Y(\cos \varphi+j \sin \varphi) \\
Y_{y x}=-Y(\cos \varphi-j \sin \varphi)
\end{array}\right\}
$$

Alternatively, substituting equations (6) into equations (2)-(5) leads to the following more explicit expressions:

$$
\begin{aligned}
& P_{x}=V_{x}^{2} G-V_{x} V_{y}\left[G \cos \left(\delta_{x}-\delta_{y}-\varphi\right)+B \sin \left(\delta_{x}-\delta_{y}-\varphi\right)\right] \\
& Q_{x}=-V_{x}^{2} B-V_{x} V_{y}\left[G \sin \left(\delta_{x}-\delta_{y}-\varphi\right)-B \cos \left(\delta_{x}-\delta_{y}-\varphi\right)\right] \\
& P_{y}=V_{y}^{2} G-V_{y} V_{x}\left[G \cos \left(\delta_{y}-\delta_{x}+\varphi\right)+B \sin \left(\delta_{y}-\delta_{x}+\varphi\right)\right] \\
& Q_{x}=-V_{y}^{2} B-V_{y} V_{x}\left[G \sin \left(\delta_{y}-\delta_{x}+\varphi\right)-B \cos \left(\delta_{y}-\delta_{x}+\varphi\right)\right]
\end{aligned}
$$

If the thyirstor controlled phase shifter is used to control the active power flowing through it, at a specified value then the Jacobin is enlarged to accommodate one additional equation. In this situation $\varphi$ enters as an extra state variable in the Jacobian equation. If the control is extended at the sending end (bus $\mathrm{x}$ ) of the phase shifter then $\mathrm{P}_{\mathrm{xy}}^{\varphi_{\mathrm{ps}}}$ is the target power to be regulated.

The set of linearised power flow equations for the TCPS is:

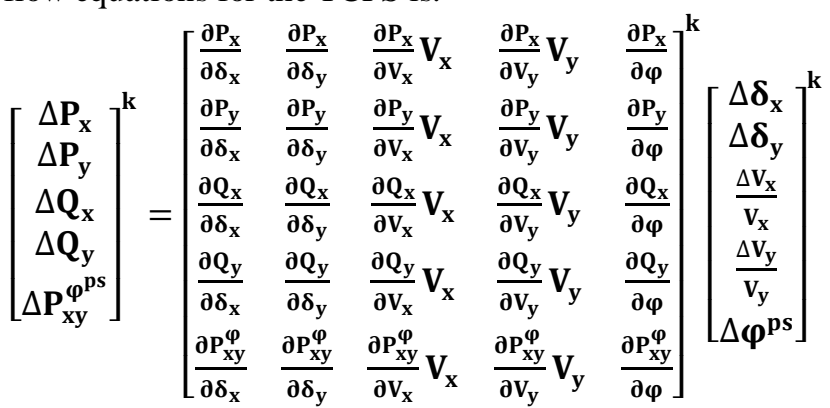

Where $\Delta \mathrm{P}_{\mathrm{xy}}^{{ }^{\mathrm{ps}}}$ given by:

$$
\Delta \mathbf{P}_{\mathrm{xy}}^{\varphi^{\mathrm{ps}}}=\mathbf{P}_{\mathrm{xy}}^{\varphi, \text { reg }}-\mathbf{P}_{\mathrm{xy}}^{\varphi^{\mathrm{ps}}}
$$

is the mismatch equation for active power flow across TCPS, $\mathrm{P}_{\mathrm{xy}}^{\varphi^{\mathrm{ps}}}$ is the calculated power as given by Equation (7). $\Delta \varphi^{\mathrm{ps}}$, given by:

$$
\Delta \varphi^{\mathrm{ps}}=\varphi^{(\mathrm{k}+1)}-\varphi^{(\mathrm{k})}
$$


is the incremental change in the thyristor controlled phase shifter angle. Superscript $\mathrm{k}$ indicates the iteration value.

\section{Formulation Of Optimal Power Flow Problem}

The OPF problem is to optimize the steady state performance of a power system in terms of an objective function while satisfying several equality and inequality constraints. Mathematically, the OPF problem can be formulated as given:

$$
\begin{array}{ll} 
& \operatorname{Min} \mathbf{F}(\mathbf{x}, \mathbf{u}) \\
\text { Subject to } & \mathbf{g}(\mathbf{x}, \mathbf{u})=\mathbf{0} \\
& \mathbf{h}(\mathbf{x}, \mathbf{u}) \leq \mathbf{0}
\end{array}
$$

Where $\mathrm{x}$ is a vector of dependent variables consisting of slack bus power, load bus voltages $V_{L}$, generator reactive power outputs $Q_{G}$ and the transmission line loadings $S_{l}$. Hence x can be expressed as given:

$$
X^{T}=\left[P_{G_{1}}, V_{L_{1}} \ldots V_{L_{N L}}, Q_{G_{1}} \ldots Q_{G_{N G}}, S_{l} \ldots . S_{l_{n l}}\right]
$$

Where NL, NG and $\mathrm{nl}$ are number of load buses, number of generators and number of transmission line respectively. $\mathrm{u}$ is the vector of independent variables consisting of generator voltages $V_{G}$, generator real power outputs $P_{G}$ except at the slack bus $P_{G_{1}}$, transformer tap settings T, and shunt VAR compensations $Q_{C}$. Hence u can be expressed as given:

$$
U^{T}=\left[V_{G_{1}} \ldots . V_{G_{N G}}, P_{G_{2}} \ldots . P_{G_{N G}}, T_{1} \ldots T_{N T}, Q_{C_{1}} \ldots Q_{C_{N C}}\right]
$$

Where NT and NC are the number of the regulating transformers and shunt compensators respectively. $\mathrm{F}$ is the objective function to be minimized, $\mathrm{g}$ is the equality constraints that represent typical load flow equations and $\mathrm{h}$ is the system operating constraints.

\section{Objective Function}

The severity of a contingency to line overload may be expressed in terms of the Severity Index, which express the stress on the power system in the post contingency period. In order to evaluate the security of the power system network a Severity Index was proposed. The objective function in the proposed OPF was selected as the minimization of the proposed Severity Index. By minimizing the value of Severity Index, it can observe an enhancement in the system security [16]. For example, in order to determine the degree of violations at the line $L_{m-n}$ the following Severity Index is proposed.

Objective function $\quad F=\min \left(S I_{m n}\right)$

$$
S I_{m n}=\frac{S_{m n}-S_{m n \max }}{S_{m n \text { max }}} \text { m,n \& NB }
$$

Where $S I_{m n}$ the Severity Index of line overloads, $S_{m n}$ is the overload flow on transmission line,$S_{\text {mnmax }}$ is the rated flow on transmission line and NB is the Set of overloaded lines.

\section{Constraints}

The OPF problem has two categories of constraints

\subsection{Equality Constraints}

These are the sets of nonlinear power flow equations that govern the power system, i.e.,

$$
\begin{gathered}
P_{G m}-P_{D m}-\sum_{n=1}^{l}\left|V_{m}\right|\left|V_{n}\right|\left|Y_{m n}\right| \cos \left(\theta_{m n}-\delta_{m}+\delta_{n}\right)=0 \\
Q_{G m}-Q_{D m}+\sum_{n=1}^{l}\left|V_{m}\left\|V_{n}\right\| Y_{m n}\right| \sin \left(\theta_{m n}-\delta_{m}+\delta_{n}\right)=0
\end{gathered}
$$

Where $P_{G m}$ and $Q_{G m}$ are the real and reactive power outputs injected at bus- $m$ respectively, the load demand at the same bus is represented by $P_{D m}$ and $Q_{D m}$, and elements of the bus admittance matrix are represented by $\left|Y_{m n}\right|$ and $\theta_{m n}$. 


\subsection{Inequality Constraints}

These are the set of constraints that represent the system operational and security limits like the bounds on the following.

\subsubsection{Generation constraints}

Generator voltages, real power outputs, and reactive power outputs are restricted by their lower and upper limits as follows:

$$
\begin{array}{cc}
\mathbf{V}_{\mathbf{G m}}^{\min } \leq \mathbf{V}_{\mathbf{G m}} \leq \mathbf{V}_{\mathbf{G m}}^{\max }, & \mathbf{m}=\mathbf{1}, \ldots, \mathbf{N G} \\
P_{G m}^{\min } \leq P_{G m} \leq P_{G m}^{\max }, & \mathbf{m}=\mathbf{1}, \ldots, \mathbf{N G} \\
Q_{G m}^{\min } \leq Q_{G m} \leq Q_{G m}^{\max }, & \mathbf{m}=\mathbf{1}, \ldots, \mathbf{N G}
\end{array}
$$

Where NG: number of generators

\subsubsection{Transformer constraints}

Transformer tap settings are bounded as follows:

$$
T_{m}^{\min } \leq T_{m} \leq T_{m}^{\max }, \quad \mathbf{m}=\mathbf{1}, \ldots, \mathbf{N T}
$$

Where NT: number of regulating transformer

\subsubsection{Shunt VAR constraints}

Shunt VAR compensations are restricted by their limits as follows:

$$
Q_{C m}^{\min } \leq Q_{C m} \leq Q_{C m}^{\max }, \mathbf{m}=\mathbf{1}, \ldots, \text { NSVC }
$$

Where NSVC: number of Shunt Var Compensators

\subsubsection{Security constraints}

These include the constraints of voltages at load buses and transmission line loadings as follows:

Where NL: number of load buses

$$
\mathrm{V}_{\mathrm{Lm}}^{\min } \leq \mathrm{V}_{\mathrm{Lm}} \leq \mathrm{V}_{\mathrm{Lm}}^{\max }, \quad \mathrm{m}=1, \ldots, \mathrm{NL}
$$

\subsubsection{Transmission lines loading}

$$
\mathrm{S}_{\mathrm{m}} \leq \mathrm{S}_{\mathrm{m}}^{\max }, \quad \mathrm{m}=1, \ldots, \mathrm{nl}
$$

Where nl: number of Transmission lines

\subsubsection{TCPS constraint}

$$
\alpha_{\mathrm{pm}}^{\min } \leq \boldsymbol{\alpha}_{\mathrm{pm}} \leq \boldsymbol{\alpha}_{\mathrm{pm}}^{\max }
$$

Where $\alpha_{\mathrm{pm}}=$ Phase shift angle of TCPS at line $\mathrm{m}$ $\alpha_{\mathrm{pm}}^{\min }, \alpha_{\mathrm{pm}}^{\max }=$ Lower and upper phase shift angle limits of TCPS at line

\section{Overview Of PSO}

From a view of social cognition, each individual in PSO can benefit from both its own experience and group findings. In its theoretical base, some factors are included. 1) Evaluation of stimulation, 2) Influences of its behavior here after by its own experience and 3) Influence to its behavior by other particles' experience.

The principle of PSO algorithm is as follows. Let $\mathrm{s}$ and $\mathrm{v}$ denote the particle's position and its corresponding velocity in search space respectively. Therefore, the $\mathrm{i}$-th particle is represented as $s_{\mathrm{i}}=\left(\mathrm{s}_{\mathrm{i} 1}, \mathrm{~s}_{\mathrm{i} 2, \ldots}, \mathrm{s}_{\mathrm{iD}}\right), \mathrm{i}=1,2, \ldots \mathrm{m}$ in the D-dimensional space. The best previous position of the $\mathrm{i}$-th particle in dimension $\mathrm{d}$-th is represented as pbest $_{\mathrm{i}}=\left(\mathrm{p}_{\mathrm{g} 1}, \mathrm{p}_{\mathrm{g} 2}, \ldots \mathrm{p}_{\mathrm{gD}}\right)$.

Each particle of the population modified its position and velocity according to the following formulas:

$$
\begin{aligned}
& v_{i d}^{k+1}=w_{i d}^{k}+c_{1} \operatorname{rand}_{1}^{*}\left(\text { pbest }_{i}-s_{i d}^{k}\right)+c_{2} \operatorname{rand}_{2}^{*}\left(\text { gbest }_{-}-s_{i d}^{k}\right) \\
& \mathbf{w}=\mathbf{w}_{\text {max }}-\frac{\mathbf{w}_{\text {max }}-\mathbf{w}_{\text {min }}}{\text { iter }_{\max }} * \text { iter } \\
& \mathbf{s}_{\mathrm{id}}^{\mathrm{k}+1}=\mathbf{s}_{\mathrm{id}}^{\mathrm{k}}+\mathbf{v}_{\mathrm{id}}^{\mathrm{k}+\mathbf{1}}
\end{aligned}
$$

Where $\mathrm{d}=1,2,3 \ldots \mathrm{D}$ is the number of members in a particle, $\mathrm{i}=1,2 \ldots \mathrm{m}$ is the number of particles in a swarm, $k$ is the number of current generation. $v_{i d}^{k}$ is the velocity of $i$-th particle in dimension d-th, $v_{i d}^{k} \epsilon[-$ $\left.\mathrm{v}_{\min }, \mathrm{v}_{\max }\right], \mathrm{w}$ is the inertia weight factor, $\mathrm{c}_{1}, \mathrm{c}_{2}$ are two positive constant parameters called acceleration coefficients,rand 1 and rand 2 are the random functions in the range $[0,1]$,gbest is the best position among all particles in the swarm and $s_{i d}^{k}$ is the current position of particle.

The first part of formula (31) is the inertia velocity of particle, which reflects the memory behaviour of particle. The second part is "cognition" part, which represents the private thinking of the particle itself. The third 
part is the "social" part, which shows the particle's behaviour from the experience of other particles in the population. The particles find the optimal solution by cooperation and competition among the particles.

\section{Adaptive PSO (APSO)}

The basic system equation of PSO (31, 32 and 33) can be considered as a kind of difference equation. Therefore, the system dynamics, that is, the search procedure, can be analyzed using eigen values of the difference equation. Actually, using a simplified state equation of PSO, Clerc and Kennedy developed CFA of PSO by eigen values. The velocity of the constriction factor approach (simplest constriction) can be expressed as follows instead of (31) and (32).

Where $\Phi$ and $\mathrm{k}$ are coefficients.

$$
\begin{aligned}
\mathbf{v}_{\mathrm{id}}^{\mathrm{k}+1} & =\mathbf{k}\left[\mathbf{v}_{\mathrm{id}}^{\mathbf{k}}+\mathbf{c}_{1}^{*} \operatorname{rand}_{1}^{*}\left(\text { pbest }_{\mathbf{i}}-\mathbf{s}_{\mathrm{id}}^{\mathbf{k}}\right)+\mathbf{c}_{2}^{*} \operatorname{rand}_{2}^{*}\left(\text { gbest }^{-s_{\mathrm{id}}}\right)\right] \\
\mathbf{k} & =2 /\left(2-\varphi-\sqrt{\Phi^{2}-4 \varphi}\right) \\
\Phi & =\mathbf{c}_{1}+\mathbf{c}_{2}, \boldsymbol{\varphi}>\mathbf{4}
\end{aligned}
$$

The following points are improved to the original PSO with Inertia Weight Approach (IWA).

1. The search trajectory of PSO can be controlled by introducing the new parameters (P1, P2) based on the probability to move close to the position of (pbest, gbest) at the following iteration.

2. The term $w v_{i d}^{k}$ of (31) is modified as (34). Using the equation, the center of the range of particle movements can be equal to gbest.

3. When the agent becomes gbest, it is perturbed. The new parameters (P1, P2) of the agent are adjusted so that the agent may move away from the position of (pbest, gbest).

4. When the agent is moved beyond the boundary of feasible regions, pbests and gbest cannot be modified.

5. When the agent is moved beyond the boundary of feasible regions, the new parameters $(\mathrm{P} 1, \mathrm{P} 2)$ of the agent are adjusted so that the agent may move close to the position of (pbest, gbest).

The new parameters are set to each agent. The weighting coefficients are calculated as follows:

$$
\mathbf{c}_{2}=\frac{2}{\mathrm{P}_{1}}, \mathbf{c}_{1}=\left(\frac{2}{\mathrm{P}_{2}}\right)-\mathbf{c}_{2}
$$

The search trajectory of PSO can be controlled by the parameters (P1, P2). Concretely, when the value is enlarged more than 0.5 , the agent may move close to the position of pbest/gbest.

$$
\mathbf{w}=\text { gbest }-\left(\left\{\mathbf{c}_{\mathbf{1}}\left(\text { pbest }_{\mathbf{i}}-\mathbf{x}\right)+\mathbf{c}_{2}(\text { gbest }-\mathbf{x})\right\} / 2+\mathbf{x}\right)
$$

Namely, the velocity of the improved PSO can be expressed as follows:

$$
v_{i d}^{k+1}=w+c_{1} \operatorname{rand}_{1}^{*}\left(\text { pbest }_{i}-s_{i d}^{k}\right)+c_{2} \operatorname{rand}_{2}^{*}\left(\text { gbest }-s_{i d}^{k}\right)
$$

The improved PSO can be expressed as follows (steps 1 and 5 are the same as PSO):

\section{Generation of initial searching points}

Basic procedures are the same as PSO. In addition, the parameters (P1, P2) of each agent are set to 0.5 or higher. Then, each agent may move close to the position of (pbest, gbest) at the following iteration.

2. Evaluation of searching points

The procedure is the same as PSO. In addition, when the agent becomes gbest, it is perturbed. The parameters (P1, P2) of the agent are adjusted to 0.5 or lower so that the agent may move away from the position of (pbest, gbest).

\section{Modification of searching points}

The current searching points are modified using the state equations (34), (39) of adaptive PSO.

\section{IPM-APSO Based Hybrid Method}

Basically, the hybrid method involves two steps. The first step employs IPM to solve OPF approximated as a continuous problem and introduced into the initial populations of APSO [17]-[19]. The second part uses APSO to obtain the final optimal solution. In initial population, all individuals (obtained from IPM) are produced randomly. The main reason for using the IPM is that it is often closer to optimal solutions than other random individuals. In the hybridization of IPM and APSO, the IPM generates best initial solutions from random initial solutions and APSO evaluate them by solving the OPF, which yields to the global optimal solutions for control variables.

The implementation steps of the proposed IPM-APSO based algorithm with TCPS can be written as follows

Step -1 Input the system data for load flow analysis

Step-2 Assume several contingencies

Step -3 Select a FACTS device and its location in the system

Step -4 At the generation Gen $=0$, set the simulation parameters of IPM-APSO parameters and randomly initialize $\mathrm{k}$ individuals within respective limits and save them in the archive. 
Step -5 For each individual in the archive, run power flow to determine load bus voltages, angles, load bus voltage stability indices, generator reactive power outputs and calculate line power flows.

Step- 6 Evaluate the penalty functions

Step -7 evaluate the objective function values and the corresponding fitness values for each individual

Step -8 Find the generation local best xlocal and global best xglobal and store them

Step- 9 Increase the generation counter Gen $=\mathrm{Gen}+1$.

Step-10 Apply the IPM-APSO operators to generate new k individuals

Step-11 For each new individual in the archive, run power flow to determine load bus voltages, angles load bus voltage stability indices, generator reactive power outputs, calculate line power flow

Step -12 Evaluate the penalty functions

Step -13 Evaluate the objective function values and the corresponding fitness values for each new individual.

Step -14 Apply the selection operator of IPM-APSO and update the individuals.

Step -15 update the generation local best xlocal and global best xglobal and store them.

Step -16 If one of stopping criterion have not been met, repeat steps 4-15. Else go to step 17

Step -17 Checking the limit violation for security constraints. If iterations reached to its max value then go to else go to step 2.

Step -18 Stop

\section{Simulation Results}

The proposed approach has been tested on the standard IEEE 30-bus test system shown in Fig.2. The system line and bus data are given in [20]. The system has six generators at buses 1, 2, 5, 8, 11, and 13 and four transformers with off-nominal tap ratio in lines 6-9, 6-10, 4-12, and 28-27. The minimum and maximum limits on control variables along with the initial operating point are given in [20].

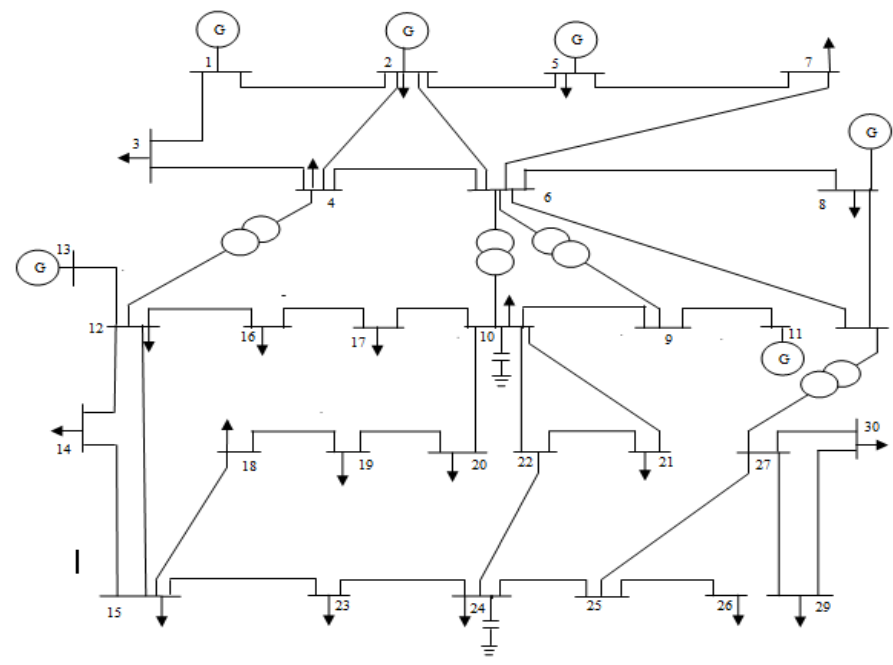

Fig.2 Single line diagram of IEEE 30 bus system

The network has total active power load of 283.4 MW. Totally there are 19 control variables which consist of six Generator Bus voltages, four Tap changing transformers and nine Shunt compensators. TCPS is installed at line connected between buses 15 and 18 with line real and reactive power settings of TCPS, Pmk = 0.10 , Qmk $=0.01$ and $-\Pi / 4 \leq \alpha_{\mathrm{pm}} \leq \Pi / 4$. The PSO parameters used for simulation are summarized in TABLE 1 .

Table 1 Optimal Parameter Settings for PSO

\begin{tabular}{|c|c|}
\hline Parameter & Value \\
\hline Population size & 20 \\
\hline Number of iterations & 150 \\
\hline Cognitive constant, $\mathrm{c}_{1}$ & 2 \\
\hline Social constant, $\mathrm{c}_{2}$ & 2 \\
\hline Inertia weight, $\mathrm{w}$ & $0.5-1.5$ \\
\hline
\end{tabular}

TABLE 2 gives the details of line outage ranking using severity index. Considering case a, when the line outage is between buses 1-2, under base case condition (without IPM-APSO and TCPS), it is observed that the lines 1-3,3-4 \& 4-6 are overloaded with line flows 190.47 MVA, 181.45MVA and 110.52 MVA respectively against their line flow limits 130 MVA ,130 MVA \& 90 MVA. In order to rectify the problem of overflows in lines IPM-APSO with TCPS has been implemented, and then the line flow limits that are violated under base case condition is rectified with the values 52.764 MVA, 49.410 MVA and 38.082 MVA respectively. 
It is observed that severity index is reduced by using IPM-APSO with TCPS technique when compared with base case severity index.

Similarly for case $b$, case $c$ and case $d$ it is noted that, IPM-APSO with TCPS based OPF maintains security of the power system network by maintaining line flows within their limits under single line contingencies and also observed that minimum severity index is obtained by IPM-APSO with TCPS method when compared with base case. Ranking is also given based on the increasing order of severity and the results that are reported in TABLE 2.

Table 2 Line Outage Ranking Using Severity Index

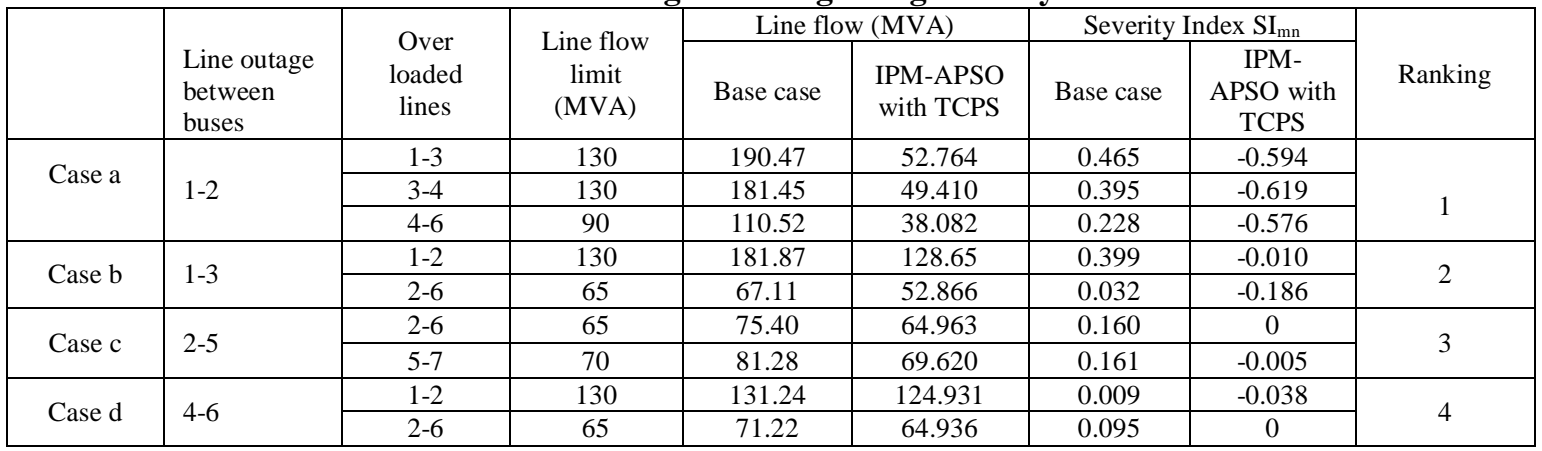

TABLE 3 presents the setting of control variables for IEEE 30-bus system for base case and IPMAPSO with TCPS at different single line outages. From the results, it is observed that all the control variables are within limits and lines are operating within the specified line limits by the application of IPM-APSO with TCPS based OPF algorithm under the occurrence of various severe network contingencies and however minimum real power loss is obtained when compared with base case.

Table 3 Control Variables Setting For IEEE 30-Bus System

\begin{tabular}{|c|c|c|c|c|c|c|c|c|}
\hline \multirow[b]{2}{*}{$\begin{array}{c}\text { Control } \\
\text { variables }\end{array}$} & \multicolumn{2}{|c|}{ Case a } & \multicolumn{2}{|c|}{ Case b } & \multicolumn{2}{|c|}{ Case c } & \multicolumn{2}{|c|}{ Case d } \\
\hline & Base case & $\begin{array}{l}\text { IPM-APSO } \\
\text { with TCPS }\end{array}$ & Base case & $\begin{array}{c}\text { IPM-APSO } \\
\text { with TCPS }\end{array}$ & Base case & $\begin{array}{l}\text { IPM-APSO } \\
\text { with TCPS }\end{array}$ & Base case & $\begin{array}{l}\text { IPM-APSO } \\
\text { with TCPS }\end{array}$ \\
\hline $\mathrm{P}_{1}$ & 193 & 52.22 & 180.58 & 130.36 & 184.09 & 162.54 & 177.76 & 162.54 \\
\hline $\mathrm{P}_{2}$ & 48.88 & 80 & 48.88 & 60.64 & 48.88 & 43.67 & 48.88 & 43.67 \\
\hline $\mathrm{P}_{3}$ & 21.75 & 35 & 21.75 & 35.00 & 21.75 & 35.00 & 21.75 & 35 \\
\hline $\mathrm{P}_{4}$ & 12.18 & 30 & 12.18 & 20.59 & 12.18 & 17.29 & 12.18 & 17.29 \\
\hline $\mathrm{P}_{5}$ & 21.51 & 50 & 21.51 & 24.98 & 21.51 & 26.82 & 21.51 & 26.82 \\
\hline $\mathrm{P}_{6}$ & 12.00 & 40 & 12.00 & 20.87 & 12.00 & 12.01 & 12.00 & 12.01 \\
\hline $\mathrm{V}_{1}$ & 1.05 & 1.05 & 1.0700 & 1.05 & 1.0700 & 1.05 & 1.0700 & 1.05 \\
\hline $\mathrm{V}_{2}$ & 1.045 & 1.0417 & 1.0538 & 1.0320 & 1.0538 & 1.048 & 1.0538 & 1.036 \\
\hline $\mathrm{V}_{3}$ & 1.01 & 1.0313 & 1.0299 & 1.0166 & 1.0299 & 1.0309 & 1.0299 & 1.0062 \\
\hline $\mathrm{V}_{4}$ & 1.05 & 1.082 & 1.0375 & 1.0438 & 1.0468 & 1.0458 & 1.0560 & 1.0708 \\
\hline $\mathrm{V}_{5}$ & 1.01 & 1.0238 & 1.0381 & 1.0151 & 1.0412 & 0.9764 & 1.0520 & 1.0089 \\
\hline $\mathrm{V}_{6}$ & 1.05 & 1.0745 & 1.0370 & 1.0736 & 1.0375 & 0.0528 & 1.0369 & 1.0186 \\
\hline $\mathrm{T}_{1}$ & ---- & 0.9906 & ---- & 0.9801 & ---- & 1.0397 & ---- & 1.0159 \\
\hline $\mathrm{T}_{2}$ & ---- & 1.0241 & ---- & 1.0084 & ---- & 1.0525 & $\begin{array}{l}--- \\
\end{array}$ & 0.9837 \\
\hline $\mathrm{T}_{3}$ & ---- & 1.012 & ---- & 1.0125 & ---- & 1.0177 & ---- & 1.0287 \\
\hline $\mathrm{T}_{4}$ & ---- & 0.9765 & ---- & 0.9649 & ---- & 1.0135 & $\begin{array}{l}--- \\
\end{array}$ & 0.998 \\
\hline $\mathrm{Q}_{\text {sh1 }}$ & ---- & 0 & ---- & 0.039 & ---- & 0.0361 & ---- & 0.0294 \\
\hline $\mathrm{Q}_{\mathrm{sh} 2}$ & ---- & 0.0004 & ---- & 0.0387 & ---- & 0.0283 & ---- & 0.0479 \\
\hline $\mathrm{Q}_{\mathrm{sh} 3}$ & ---- & 0.0353 & ---- & 0.0478 & ---- & 0.0284 & ---- & 0.0613 \\
\hline $\mathrm{Q}_{\mathrm{sh} 4}$ & ---- & 0.0673 & ---- & 0.0751 & ---- & 0.0563 & ---- & 0.046 \\
\hline $\mathrm{Q}_{\mathrm{sh} 5}$ & ---- & 0.0318 & ---- & 0.0651 & $\begin{array}{l}--- \\
\end{array}$ & 0.0889 & ---- & 0.0408 \\
\hline $\mathrm{Q}_{\text {sh6 }}$ & ---- & 0.0479 & ---- & 0.073 & ---- & 0.0595 & ---- & 0.0175 \\
\hline $\mathrm{Q}_{\text {sh7 }}$ & ---- & 0.0442 & ---- & 0.0548 & ---- & 0.0584 & ---- & 0.054 \\
\hline $\mathrm{Q}_{\text {sh8 }}$ & ---- & 0.1 & ---- & 0.0548 & ---- & 0.0529 & ---- & 0.0558 \\
\hline $\mathrm{Q}_{\text {sh9 }}$ & ---- & 0.0205 & ---- & 0.0708 & $\begin{array}{l}--- \\
\end{array}$ & 0.0486 & ---- & 0.0489 \\
\hline $\operatorname{Cost}(\$ / \mathrm{hr})$ & 857.94 & 969.3728 & 815.7052 & 828.7239 & 827.5252 & 828.6864 & 806.2758 & 808.6453 \\
\hline Loss(MW) & 3.0932 & 0.2592 & 0.135 & 0.0904 & 0.1701 & 0.1393 & 0.4437 & 0.1393 \\
\hline
\end{tabular}

\section{Conclusion}

This paper presents an improved, efficient and reliable IPM-APSO with TCPS algorithm for solving Optimal Power Flow problem under occurrence of various single line contingencies. The proposed method is tested on IEEE-30 bus system and the simulation results are reported.

From the results it can be concluded that Severity index, that is calculated indicates how much severe a possible line outage is and the severity of each line outage in the system. The severity index with highest value indicates the severity of that particular line outage and also indicates that it has got maximum chances of making 
system parameters to operate beyond the operating limits. The IPM-APSO with TCPS based Optimal Power Flow algorithm mitigates severity index and shows better performance under critical conditions. The results show the effectiveness and robustness of the proposed algorithm in order to solve OPF problem.

\section{References}

[1]. Atiya naaz, L.Sayyed, Pramod M.Gadge and Ruhi Uzma Sheikh,"Contingency Analysis and Improvement of Power System Security by locating Series FACTS Devices “TCSC and TCPAR' at optimal location”, IOSR Journal of Electrical and Electronics Engineering e-ISSN: 2278-1676, P-ISSN: 2320-3331, pp19-27.

[2]. M.R.Iravani and D.Maratukulam,"Review of semiconductor controlled (static) phase shifters for power system applications", IEEE Trans. on Power Systems, vol. 9, no. 4, pp. 1833-1839, 1994.

[3]. Wei Shao and Vijay Vittal,'LP-Based OPF for Corrective FACTS Control to Relieve Overloads and voltage violations", IEEE Trans.on Power Systems, vol.21, no.4, November 2006.

[4]. M. Huneault and F.D. Galiana,"A Survey of the Optimal Power Flow Literature”, IEEE Trans. on Power Systems, Vo1.6, No. 2, May 1991.

[5]. James A.Momoh, M.E.EI-Hawary and Rambabu Adapa, “A Review of Selected Optimal Power Flow Literature to 1993 Newton Linear Programming and Interior Point Methods”., IEEE Trans. on Power Systems, vol.14, no.1, February 1999.

[6]. Sergio Granville and CEPEL,"Optimal Reactive Dispatch through Interior Point Method", IEEE Trans .on Power Systems, vol.9, no.1, February 1999.

[7]. Ding Xiaoying, Wang Xifan, Song Yonghua and Geng Jian,"The Interior Point Branch and Cut method for Optimal Power Flow", IEEE 2002; 0-7803-7459.

[8]. Kyoung-shin Kim, Leen -Hark Jung and Seung -chul ,"Security Constrained Economic Dispatch using Interior Point Method”, International conference on Power system Technology,2006.

[9]. R.V.Amarnath and Dr.N.V.Ramana,"State of Art in optimal power flow solution methodologies“, Journal of theoretical and Applied Information Technologies $31^{\text {st }}$ August 2011.vol 30, no 2

[10]. Anastasias,G.Bakirtzis, pandel N,Biskas and Christoforos, “Optimal power flow by enhanced Genetic Algorithm”.IEEE Trans on Power Systems vol 17,no 2 May 2002.

[11]. M.Sailaja Kumari, G.Priyanka and M.Sydulu "Comparison of Genetic Algorithms and Particle Swarm Optimization for Optimal Power Flow Including FACTS devices” IEEE 2007; 978-1-4244-2190.

[12]. Yuryevich and Jawing KP.”Evolutionary programming based optimal power flow algorithm". IEEE Trans Power Syst 1999; 14(4):1245-50.

[13]. Bhavna Sharma and Mandaree panjit "Security Constrained Optimal Power Flow employing Particle Swarm Optimization".IEEE 2012, 978-1-4673-1515.

[14]. Yamille del valle,Ganesh Kumar and Venayagamoorthy“Particle Swarm Optimization :Basic concepts, Variants and Applications in Power Systems",IEEE Trans on Evalutionary Computation ,vol 12,no 2,April 2008.

[15]. Hc Leung and Dylan Dah-chuan Lu“Particle Swarm Optimization for OPF with consideration of FACTS devices”.IEEE 2011:9781-61284-972.

[16]. B.Rajanarayana Prusty, Bhagabati Prasad Pattnaik, Prakas Kumar Pandey and A.Sai Santhosh "Power System Security Analysis". International Journal of Scientific \&Engineering Research, vol5, issue 5, May-2014 ISSN 2229-5518.

[17]. N.M. Tabatabaei, Gh. Aghajani,N.S. Boushehri and S. Shoarinejad, "Optimal location of Facts devices using Adaptive Particle Swarm Optimization mixed with Simulated Annealing”. International Journal on Technical and Physical Problems of Engineering (IJTPE) Published by International Organization on TPE (IOTPE), June 2011, Issue 7, Volume 3, Number 2.

[18]. P.k.Tripathi, Sanghamitra, Bandyopadhyay, and S.K.Pal, "Adaptive Multi-objective Particle Swarm Optimization Algorithm", IEEE 2007:1-4244-1340.

[19]. S.he, J.Y.wen, E.prempain,.H.wu,J.Fitch and S.Mann "“An Improved Particle Swarm Optimization for Optimal Power Flow”. International conference on power system technology-powercon 2004.

[20]. K Y Lee, Y M Park and J L Ortiz,"A United Approach to Optimal Real and Reactive Power Dispatch". IEEE Trans. On Power Apparatus and System, vol 104, 1985, pp1147-1153.

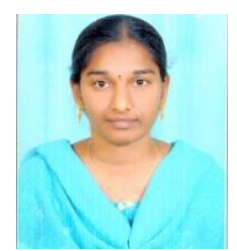

K.SriLakshmi Lavanya is pursuing her M.Tech (Power System Automation and Control) in the Department of Electrical \& Electronics Engineering, from P.V.P.Siddhartha Institute of Technology, Vijayawada, Andhra Pradesh, India. She obtained B.Tech degree in Electrical and Electronics Engineering from Lakireddy Balireddy College of Engineering in the year 2010.

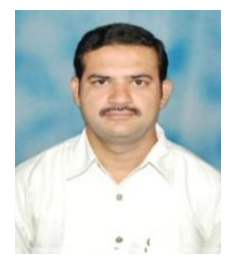

C.Kumar is currently working as Associate Professor in the Department of Electrical \&Electronics Engineering, P.V.P.Siddhartha Institute of Technology, Vijayawada, Andhra Pradesh, India. He obtained M.Tech from J.N.T.U.College of Engineering, Hyderabad and doing Ph.D in the area of power system security at J.N.T.University, Anantapur. His areas of interest are Power System Security, OPF techniques and FACTS.

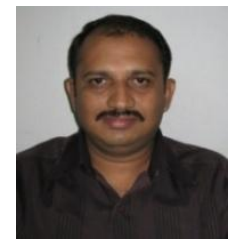

Dr.Ch.Padmanabha Raju is currently working as Professor in the Department of Electrical \& Electronics Engineering, P.V.P.Siddhartha Institute of Technology, Vijayawada, Andhra Pradesh, India. He obtained Ph.D from J.N.T.University, Kakinada .His areas of interest are Power System Security, OPF techniques, Deregulation and FACTS. 